\title{
Restoring ATBF: Dreaming the impossible dream?
}

Commentary on: Subcutaneous adipose tissue blood flow and vasomotion in morbidly obese patients: Long term effect of gastric bypass surgery, by M. Rossi, M. Nannipieri, M. Anselmino et al. Vol. 51(3), 2012, pp. 159-167

Richard Sotorník, Maude Gagnon-Auger, Pascal Brassard and Jean-Luc Ardilouze* Diabetes and Metabolism Research Group, Division of Endocrinology, Department of Medicine, Centre Hospitalier Universitaire de Sherbrooke, Sherbrooke, QC, Canada

\section{Dear Sir:}

Knowledge of the longterm effect of weight loss on adipose tissue blood flow (ATBF) is scarce. We read with great interest the paper by Rossi et al. [3], reporting the results of a study using laser-Doppler flowmetry (LDF), where baseline thigh ATBF was lower in morbidly obese subjects compared to age- and sex-matched controls (4.8 vs. 79.9 perfusion units /PU). These results confirm several previous studies [4]. After one-year follow-up subsequent to Roux-en Y gastric bypass (RYGB) in obese patients, they observed a meaningful weight loss $(-40 \mathrm{~kg}$, e.g. $-28 \%)$. This weight loss was associated with a slight but nevertheless significant increase in ATBF (up to 10.0 PU) although patients remained obese.

The main limitations of the Rossi et al. study are linked to the inherent drawbacks of the LDF method, relating mainly to calibration, multiple Doppler shifts, tissue optical properties, motion artefacts, biological zero and impossibility to express the results in absolute values [2]. The gold-standard for ATBF measurement is the 133 xenon wash-out method, which should be combined with LDF in order to validate data obtained therewith [1]. Anemia, a frequent complication of RYGB, and antihypertensive drugs, which may influence adipose tissue perfusion [4], could also interfere with LDF measurements. However, no indication relating to these specific issues was provided in the paper. Additionally, the chosen site of measurement stands out as another scientific issue, because subcutaneous adipose tissue in the thigh is known to be much less active than in abdomen [4].

Rossi et al.'s conclusion to the effect that the slight increase in ATBF, observed one year after RYGB, is rather negligible, is sustained by another study [5] reporting on ATBF following a very low-calorie diet

\footnotetext{
${ }^{*}$ Corresponding author: Jean-Luc Ardilouze, Diabetes and Metabolism Research Group, Division of Endocrinology, Department of Medicine, Centre Hospitalier Universitaire de Sherbrooke, Université de Sherbrooke 3001 12th Ave N., Sherbrooke, QC, J1H 5N4, Canada. E-mail: jean-luc.ardilouze@ usherbrooke.ca.
} 
(VLCD) in obese subjects. Although the former and the latter [3 vs. 5] diverged with respect to the sites of measurement of ATBF (thigh vs. abdomen), the magnitude of weight loss ( $-40 \mathrm{vs} .-11 \mathrm{~kg}$ ), baseline BMI ( $46 \mathrm{vs.} 33 \mathrm{~kg} / \mathrm{m}^{2}$ ) and the time scale (1 year vs. 6 weeks), there was no noteworthy change in ATBF per gram of adipose tissue observed in the VLCD study.

Therefore, improving ATBF remains a challenge. ATBF after persistent weight loss and a long period of metabolic stabilization could however be very distinct from that observed 1 year or only 6 weeks following a major surgical procedure such as RYGB and/or a drastic diet modification. Indeed, improvements of two major factors regulating ATBF [4], insulin resistance and the sympathetic nervous system, which both affect ATBF [4] still continue progressing well beyond these periods. Could further weight loss lead to greater ATBF improvement? This commands such pronounced weight loss, that it remains virtually unattainable in massively obese people. Therefore, because of the ATBF gap between obese and control subjects at baseline, and the huge improvement in BMI after RYGB, any further ATBF improvement seems highly unlikely. The study suggests, on the contrary, that ATBF is substantially abolished at a certain degree of obesity and that further adipose tissue expansion has little impact on ATBF.

Rossi et al.'s paper is interesting despite the preliminary nature of the results. The LDF method is promising and further prospective studies are needed to investigate emaciation-induced improvement of ATBF in moderately obese subjects able to reach a BMI under $25 \mathrm{~kg} / \mathrm{m}^{2}$.

\section{Acknowledgments}

RS is a Department of Medicine Scholar. MAG is supported as a PhD student by the Fonds de la recherche en santé du Québec (FRSQ). JLA is a Canadian Institutes for Health Research scholar (2006 New Investigator award). All authors are members of the FRSQ-funded Clinical Research Centre Étienne-Le Bel of the CHUS. The authors comply with the Ethical Guidelines for Publication in Clinical Hemorheology and Microcirculation as published on the IOS Press website and in Volume 44, 2010, pp. 1-2 of this journal.

\section{References}

[1] E. Martin, P. Brassard, M. Gagnon-Auger, P. Yale, A.C. Carpentier and J.L. Ardilouze, Subcutaneous adipose tissue metabolism and pharmacology: A new investigative technique, Can J Physiol Pharmacol 89 (2011), 383-391.

[2] V. Rajan, B. Varghese, T.G. van Leeuwen and W. Steenbergen, Review of methodological developments in laser Doppler flowmetry, Lasers Med Sci 24 (2009), 269-283.

[3] M. Rossi, M. Nannipieri, M. Anselmino, D. Guarino, F. Franzoni and M. Pesce, Subcutaneous adipose tissue blood flow and vasomotion in morbidly obese patients: Long term effect of gastric bypass surgery, Clin Hemorheol Microcirc 51 (2012), $159-167$.

[4] R. Sotornik, P. Brassard, E. Martin, P. Yale, A.C. Carpentier and J.L. Ardilouze, Update on adipose tissue blood flow regulation, Am J Physiol Endocrinol Metab 302 (2012), E1157-1170.

[5] A.P. Viljanen, R. Lautamäki, M. Järvisalo, R. Parkkola, R. Huupponen, T. Lehtimäki, T. Rönnemaa, O.T. Raitakari, P. Iozzo and P. Nuutila, Effects of weight loss on visceral and abdominal subcutaneous adipose tissue blood-flow and insulin-mediated glucose uptake in healthy obese subjects, Ann Med 41 (2009), 152-160. 